\title{
A hybrid model for China's power grid investment demand forecasting based on variational mode decomposition, regularized extreme learning machine and support vector machine
}

\author{
Qianqian $\mathrm{Wu}^{1}$, Shaowen $\mathrm{Zhu}^{1}$, Jinchao $\mathrm{Li}^{1,2, *}$, Wenjun $\mathrm{Chen}^{1,2}$, Yunna $\mathrm{Wu}^{1,2}$ \\ ${ }^{1}$ School of Economics and Management, North China Electric Power University, Beijing, China \\ ${ }^{2}$ Beijing Key Laboratory of New Energy and Low-Carbon Development, North China Electric Power University, Beijing, China
}

\begin{abstract}
With the continuous maturity of China's power grid as well as the advancement of electricity market reform in China, accurate and efficient investment decision has become an inevitable requirement of power grid enterprises. However, China's Power grid investment demand has complicated nonlinear and non-stationary characteristics due to it's complex causes of formation, thus make it hard to be forecasted. Aiming at this problem, this paper puts forward a novel hybrid VMD-RELMLOO-PSOSVM forecasting model based on variational mode decomposition (VMD), leave-one-out cross validation error based optimal regularized extreme learning machine (RELM-LOO) and support vector machines optimized by particle swarm optimization algorithm (PSO-SVM). Firstly, the VMD method is employed to decompose the original power grid investment data sequence into several modes which have specific sparsity properties while producing main signal. Then, according to the different characteristics of each subsequence, the RELM-LOO and PSO-SVM model will be used to forecast different modes, respectively; Next, the prediction results of all modes are aggregated to obtain the final prediction results of China's power grid investment demand. Finally, this paper predicts China's power grid investment demand from 2018 to 2020 under 5 different scenarios based on the proposed VMD-RELMLOO-PSOSVM hybrid forecasting model.
\end{abstract}

\section{Introduction}

In recent years, the advancement of electricity market reform in China has greatly improved marketization degree of power industry. Against this background, power grid enterprises have to continuously improve their decision-making quality and efficiency to enhance enterprise's operational efficiency and market competitiveness. For power grid enterprises, power grid investment demand decision is critical for enterprises' development due to it's huge investment amount along with long payback period [1]. However, China's power grid investment demand is influenced by many factors such as economic development, price level, national policy and other uncertainties, complex causes of formation bring China's power grid investment demand with complicated nonlinear and non-stationary characteristics. So, in this paper, we devote to put forward an accurate and effective forecasting model for China's power grid investment, thus to improve the investment decision-making quality and efficiency of power grid enterprises.

The study on power grid investment forecasting model is still less. ZHAO Huiru, etc. [2] proposes a cointegration theory and error correction model to forecast the power grid investment demand, this model finds out the logarithmic linear relation between the power grid investment and influencing factors of the power grid investment; However, the power grid investment has complicated nonlinear nonstationary characteristics, the proposed forecasting model in this paper hardly excavate the internal laws and characteristics of the power grid investment. Shuyu Dai,etc. [1] proposes a hybrid support vector machine optimized by differential evolution algorithm and grey wolf optimization algorithm(DEGWO-SVM) to forecast China's power grid investment based on the historical data from 1990 to 2016; The support vector machine can effectively process the complex nonlinear data and brilliant parameters optimization ability of the intelligent algorithm, the DEGWO-SVM model has an outstanding prediction performance for China's power grid investment. Jinchao $\mathrm{Li}$, etc. [3] utilize the empirical mode decomposition(EMD) method to decompose the original power grid investment data series into several intrinsic mode functions and a residual component which contain different characteristics of the original power grid investment data series, respectively; These subsequences decomposed have simpler frequency feature and stronger correlations which are easier to build the prediction model compared to the original data series, thus further improve the prediction accuracy prominently.

\footnotetext{
* Corresponding author: lijc@ncepu.edu.cn
} 
In this paper, a new hybrid intelligent method based on variational mode decomposition (VMD) is employed to forecast China's power grid investment based on the history data series from 1990 to 2017. First, the VMD method is employed to decompose China's power grid investment into several modes which have specific sparsity properties. Then, the time series of each decomposed mode will be forecasted by appropriate methods. Finally, the forecasting results of all decomposed modes will be aggregated to obtain the final forecasting result of China's power grid investment. In view of the lack of the sample numbers of China's power grid investment and enhancing the generalization ability of the prediction model to further improve the accuracy and effectiveness of the VMD-based power grid investment forecasting model, the support vector machine optimized by particle swarm optimization (PSO-SVM) algorithm and the leave-one-out cross validation error based optimal regularized extreme learning machine (RELM-LOO) algorithm will be employed to forecasting the decomposed modes.

The structure diagram of the VMD-RELM-PSOSVM hybrid forecasting model is shown in Figure 1.

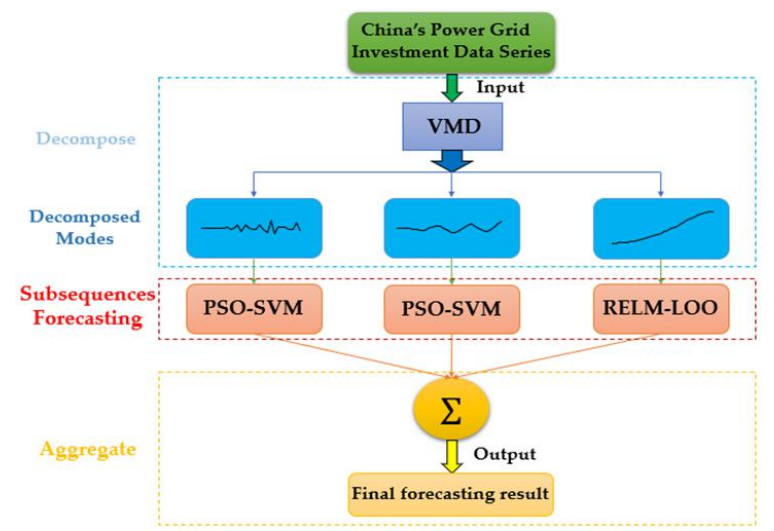

Fig. 1. Structure diagram of the VMD-RELMLOO-PSOSVM hybrid forecasting model.

The rest of this article is arranged as follows: Section 2 introduces the methodologies; Section 3 carries out empirical analysis to verify the validity of the proposed model for the power grid investment prediction in China; Section 4 forecasts the power grid investment in China from 2018 to 2020 under 5 different scenarios; Section 5 summarizes the whole paper.

\section{Methodology}

\subsection{Variational mode decomposition}

Variational mode decomposition (VMD) was proposed by Dragomiretskiy and Zosso [4] in 2014, it is a newly developed multiresolution technique for adaptive and non-recursive signal decomposition. The purpose of the VMD is to decompose an input signal $y$ into $k$ discrete number of sub-signals (modes), where each mode has limited bandwidth in spectral domain. And, each mode $y_{k}$ is required to be mostly compact around a center pulsation $\omega_{k}$ determined along with the decomposition.

\subsection{Regularized extreme learning machine optimized by leave-one-out cross validation (RELM-LOO)}

A simple and efficient learning algorithm for singlehidden layer feedforward neural networks (SLFNs) called extreme learning machine (ELM) which was proposed by Huang et al [5]. However, since ELM doesn't consider heteroskedasticity in real applications, its performance will be affected seriously when outliers exist in the dataset. In order to address these drawbacks, regularized extreme learning machine based on structural risk minimization principle and weighted least square was proposed by Deng et al [6]. For the regularized ELM described in [6], the search for the optimal regularization parameter is important, as different regularization parameter has different generalization performance. Aiming at this problem, Cao et al [7] proposed a LOO error based optimal regularized ELM for classification problem, this method can achieve better classification performance by employing the leave-oneout cross validation (LOO) approach to find out the best regularization parameter of the regularization extreme learning machine.

\section{3 support vector machine optimized by particle swarm optimization (PSO-SVM)}

The support vector machines (SVMs) were proposed by Vapnik. It is a statistical learning algorithm based on VC (Vapnik-Chervonenkis) dimension theory and structural risk minimization (SRM) principle.SVM has a strong capacity for processing nonlinear data [8]. The basic principle of support vector machines is to find a nonlinear mapping function $\phi(x)$ to make the linear inseparable data $\mathrm{x}$ in the low-dimensional feature space projected into the high-dimensional feature space $\mathrm{F}$ to make it a linear separable problem.

Particle swarm optimization (PSO) was first proposed by Kennedy and Eberhart in 1995. It is an emerging population-based meta-heuristic that simulates social behavior such as birds flocking to a promising position to achieve precise objectives in a multidimensional space. Like evolutionary algorithms, PSO searches for optimal solution in solution space using population of particles. It can be used for parameter determination and feature selection in the SVM. The operation steps of PSO-SVM are described in detail in [9].

\section{Case study}

In this section, we establish the hybrid VMDRELMLOO-PSOSVM model for China's power grid investment prediction. To further validate the effectiveness and accuracy of the proposed VMDRELM-PSOSVM hybrid forecasting model. The VMDPSOSVM model, VMD-RELMLOO model, EMD-based hybrid forecasting models and the single regularized extreme learning machine model as well as PSO-SVM model are set as benchmark models of the proposed 
VMD-RELM-PSOSVM model. The mean absolute percentage error (MAPE) and the root mean square error (RMSE) will be the evaluation criteria for quantitative analysis of different forecasting models.

\subsection{VMD-RELMLOO-PSOSVM hybrid forecasting model for China's power grid investment}

Since the VMD requires a predetermined number of decomposition $\mathrm{k}$, the latter is varied depending on the number of IMFs obtained by EMD [10]. If the number of IMFs is $\mathrm{d}$, then the parameter $\mathrm{k}$ used to determine the number of decomposition components to obtain by EMD is $d-3, d-2, d-1, d, d+1, d+2, d+3$.

In this paper, we determine the value of $\mathrm{k}$ according to the MAPE and RMSE of different VMD-RELMLOOPSOSVM model which have different decomposition value $\mathrm{k}$. Table 1 shows the calculation results under different models with different $\mathrm{k}$ value.

Table 1. MAPE and RMSE of different VMD-RELMLOOPSOSVM model with different $k$ value.

\begin{tabular}{|c|c|c|c|c|}
\hline Indicator & $\boldsymbol{k}=\mathbf{3}$ & $\boldsymbol{k}=\mathbf{4}$ & $\boldsymbol{k}=\mathbf{5}$ & $\boldsymbol{k}=\mathbf{6}$ \\
\hline MAPE & $3.3 \%$ & $3.7 \%$ & $3.9 \%$ & $4.8 \%$ \\
\hline RMSE & 120.7 & 126.1 & 139.3 & 172.2 \\
\hline
\end{tabular}

According to the calculation results, we can conclude that when $k=3$, the proposed VMD-RELMLOOPSOSVM has the best forecasting performance, so in this paper, we decompose China's power grid investment into 3 modes. Figure 2 gives the decomposed result of China's power grid investment by VMD method.

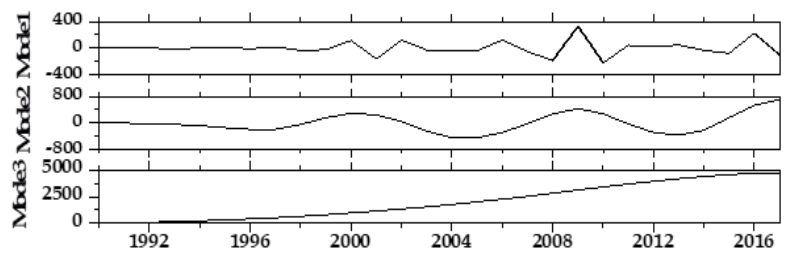

Fig. 2. VMD decomposition diagram.

As we can see from Figure 2, mode 1 has a significant stochastic characteristic while mode 2 has a significant periodic characteristic. Time series prediction method is suitable for processing stationary data series which have stochastic or periodic characteristics. Traditional time series prediction method like ARIMA has a major defect for forecasting nonlinear data series while neural network algorithm and support vector machine algorithm have great properties to process nonlinear data series. However, China's power grid investment data series is relative short, the data samples of mode 1 and mode 2 are too small for the training of the neural network while support vector machine has a great property to process problems with small number of samples. So in this paper, the support vector machine optimized by particle swarm optimization (PSO-SVM) algorithm will be used to forecast mode1 and mode 2 which have significant nonlinear and high frequency characteristics.

As we can see from Figure 2, mode 3 is a smooth increasing curve which has a significant trend characteristic. And we use the regularized extreme learning machine (RELM) combined with leave one out cross validation to forecast mode 3 . Mode 3 reveals the basic development trend of China's power grid investment, it is directly affected by the development level of China's economic and social as well as electricity production and consumption, so in this paper, we select GDP growth rate, the share of secondary industry, urbanization rate, total electricity installed capacity, total electricity consumption, share of nonfossil energy power generation installed capacity and population as inputs of the RELM-LOO model for the forecasting of mode 3 .

\subsection{Results and discussion}

In this section, we will analyze the effectiveness and accuracy of the proposed VMD-RELMLOO-PSOSVM model based on the forecasting results presented in Table 2. And three conclusions can be found based on these forecasting results.

First, we compare the forecasting results of the VMD-RELMLOO and VMD-PSOSVM model with the proposed VMD-RELMLOO-PSOSVM model. We can find out that the proposed VMD-RELMLOO-PSOSVM model has better APE performance than the other two VMD-based forecasting models, these results confirm the effectiveness and accuracy of the proposed VMDRELMLOO-PSOSVM model compared to other VMDbased models.

Second, the forecasting results of EMD-based models will be compared with VMD-based models. All the VMD-based models have better forecasting performance compared with their corresponding EMD-based model. This confirms the superiority of VMD method compared with EMD method for China's power grid investment.

Finally, the forecasting results of RELMLOO and PSOSVM model will be compared with VMDRELMLOO-PSOSVM model. Both of them have bad forecasting accuracy in extremes while the proposed VMD-RELMLOO-PSOSVM model has primely solved this problem. This further confirms the robustness and effectiveness of the proposed VMD-RELMLOOPSOSVM model. 
Table 2. Calculation results of MAPE and RMSE of different forecasting models.

\begin{tabular}{|c|c|c|c|c|c|c|c|c|}
\hline Year & VRS & VR & VS & ERS & ER & ES & R & S \\
\hline MAPE & 0.033 & 0.052 & 0.043 & 0.068 & 0.082 & 0.071 & 0.067 & 0.069 \\
\hline RMSE & 120.7 & 183.1 & 126.9 & 241.6 & 291.3 & 222.9 & 264.4 & 349.1 \\
\hline
\end{tabular}

\section{Scenario Analysis}

In this section, China's power grid investment demand from 2018 to 2020 under five different scenarios will be forecasted based on the proposed VMD hybrid forecasting model. First, the PSO-SVM model will be employed to forecast the high frequency model and mode 2 components separately in advance for three years. Then, the mode 3 component which has a trend characteristic will be forecast by RELM-LOO model under different scenarios. Finally, the forecasting results of the power grid investment demand in the next three years will be obtained by aggregating the forecasting results of all the modes.

According to the 13th Five-Year plan (2016-2020) which was published by Chinese government in 2016, the target of the GDP growth was set to more than $6.5 \%$ and the urbanization rate will reach $60 \%$ by the end of 2020, so in this paper the GDP growth rate under different scenarios will be set to $6.5 \%, 6.6 \%, 6.7 \%, 6.8 \%$ and $6.9 \%$ respectively in the next three years. And the urbanization rate in the next three year will be set to a fixed value under all scenarios, reaching 59\%, 59.5\% and $60 \%$, respectively. And the share of secondary industry will be set to $40 \%, 39 \%$, and $38 \%$ in the next three years, respectively.

According to the "Electric power development planning in 13th Five-Year" (2016-2020), the total installed capacity of power generation will increase at an annual growth rate of $5.5 \%$ while the target share of installed capacity of non-fossil energy was set to $39 \%$ by the end of 2020, so in this paper the growth rate of total installed capacity of power generation will set to $5.5 \%$ from 2018 to 2020 under different scenarios, and the share of installed capacity of non-fossil energy will be set to $37 \%, 38 \%$ and $39 \%$ in the next three years, respectively. Meanwhile, the growth rate of total electricity consumption was set to $3.6 \%-4.8 \%$ in the planning, so the annual growth rate of total electricity consumption under different scenarios will be set to $3.6 \%, 3.9 \%, 4.2 \%, 4.5 \%$ and $4.8 \%$ in the next three years, respectively. Refer to the 13th Five-Year national family planning development plan, we set the growth rate of population to $0.6 \%$ from 2018 to 2020 .

The forecasting results of China's power grid investment under different scenarios are shown in Figure 3.

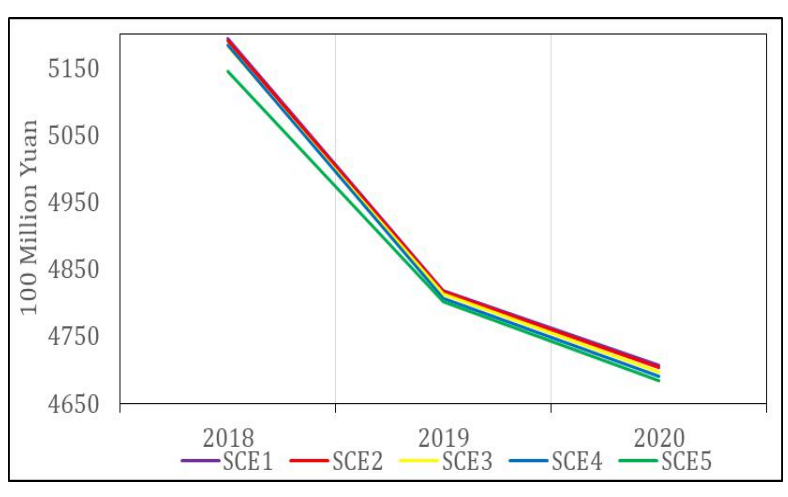

Fig. 3. Forecasting results of China's power grid investment from 2018 to 2020 under different scenarios.

Compared with the rapid growth of the power grid investment from 2012 to 2016, the forecasting results indicate that the power grid investment will not substantially increase in the next three years. In contrast, the downward trend of the power grid investment demand in the next three years would be happened.

\section{Conclusion}

In order to improve power grid enterprises' investment decision-making quality and efficiency, this paper puts forward a newly hybrid VMD-RELM-PSOSVM forecasting model for China's power grid investment demand. Firstly, the VMD method is employed to decompose the original power grid investment into three modes. Then mode 1 and mode 2 with high frequency characteristics are forecasted by PSO-SVM models while mode 3 which has a trend characteristic will be forecasted by RELM-LOO model. Then, the prediction results of different modes are summing up to get the final power forecasting results of China's power grid investment demand. Next, the VMD-PSOSVM model, VMD-RELMLOO model, the previously popular EMDbased forecasting models and single RELM-LOO model as well as PSO-SVM model are set as benchmark models of the proposed VMD-RELM-PSOSVM hybrid forecasting model; The RMSE and MAPE calculation results of different forecasting models suggest that the VMD-RELM-PSOSVM hybrid model has a better prediction performance than all the benchmark models. Finally, we forecast China's power grid investment from 2018-2020 under five different scenarios; The forecasting results indicate that China's power grid investment will decline in the next three years. 


\section{Acknowledgments}

This work has been supported by the Fundamental Research Funds for the Central Universities (no. 2018ZD14) and the Science and Technology Project of SGCC "Research on lean investment optimization method based on benefit analysis of the whole process of power grid".

\section{References}

1. S.Y. Dai, D.X. Niu, Y.R. Han. Applied Sciences, 8, $636(2018)$

2. H.R. Zhao, L. Yang, C.J. Li, X. Ma. Power System Technology, 35, 193-198 (2011)

3. J.C. Li, S.W. Zhu, Q.Q. Wu, P.F. Zhang. MATHEMATICAL PROBLEMS IN ENGINEERING (2018)

4. K. Dragomiretskiy, D. Zosso. IEEE Trans Signal Process, 62, 531-44 (2014)
5. G.B. Huang, Q.Y. Zhu, C.K. Siew. Neurocomputing, 70, 489-501 (2006)

6. W. Deng, Q. Zheng, L. Chen. 2009 IEEE Symposium on Computational Intelligence and Data Mining, Nashville, TN, 389-395 (2009).

7. J.W. Cao, K. Zhang, M.X. Luo, C. Yin, X.P. Lai. Neural Networks, 81, 91-102 (2016).

8. V. Vapnik, S. Golowich, A. Smola. Adv. Neural Inf. Process. Syst, 9, 281-287 (1996).

9. A. Selakov, D. Cvijetinović, L. Milović, S. Mellon, D. Bekut. Applied Soft Computing, 16, 80-88 (2014).

10. R. Zhang, Z.Y. Dong, Y. Xu, K. Meng, K.P. Wong. IET GENERATION TRANSMISSION \& DISTRIBUTION, 7, 391-397 (2013). 